\title{
МАТЕМАТИЧЕСКИЕ МОДЕЛИ ОЦЕНКИ СОСТОЯНИЯ ВЫПУСКНИКОВ ВУЗА
}

\author{
О. В. Курипта, Ю. В. Черная, Е. В. Паршина \\ Воронежский государственный технический университет
}

Поступила в редакцию 02.03.2018 г.

\begin{abstract}
Аннотация. В работе предлагаются модели оценки состояния выпускников вуза на этапе их отбора на вакансию. При разработке математической модели для определения соответствия выпускника вуза вакансии организации авторами предлагается комплексный подход, учитывающий критериальные оценки каждого из показателей потенциала выпускника и реализующий механизм компенсирования недостатка одного показателя существенным превышением порогового уровня другого.

Ключевые слова: оценка соответствия выпускника, критерии оценивания выпускника, математические модели, модель оптимизации, ресурсная задача.

Annotation. The paper proposes models for assessing the status of graduates at the stage of their selection for a job. When developing a mathematical model to determine the compliance of a graduate of a higher education institution with the vacancy of an organization, the authors propose an integrated approach that takes into account the criteria estimates of each of the indicators of the graduate's potential and the real mechanism of compensating for the lack of one indicator by a significant excess of the threshold level of another.

Keywords: evaluation criteria of the graduate, mathematical models, assessment of compliance of the graduate, the optimization model of the resource task.
\end{abstract}

Значение своевременного и комплексного анализа динамики трудовых ресурсов сложно переоценить, поскольку он представляет действенную методологическую и информационную базу для разработки мероприятий, проводимых в целях повышения эффективности кадровой политики. На таком анализе базируются основы оптимального принятия решений по кадровым, производственным, финансовым, инвестиционным и прочим вопросам, способствующим процветанию предприятия, а значит и социально-экономическому росту в целом всего региона.

Существенную роль в процессе регулирования и эффективного использования трудовых ресурсов играет рынок труда. Исследование публикаций и имеющейся статистической информации показало, что сегодня отсутствуют комплексные методы исследования проблем управления трудовыми ресурсами, особенностей их формирования и эффективного применения.

() Курипта О. В., Черная Ю. В., Паршина Е. В., 2018
В то же время, известные методы управления и планирования в данной сфере утратили свою актуальность в современных условиях переходной экономики или позволяют не в полной мере оценить данный аспект [1]. Нестабильные цены, сложные условия рыночных отношений, существование определенной вероятности невыполнения законодательных актов по регламентированию распределения выпускников привело к необходимости каждого вуза своевременно реагировать на все изменения, затрагивающие региональный рынок труда, а также к необходимости модификации своей поведенческой тактики и стратегии при распределении выпускников. При этом возникает потребность учета непрерывно меняющихся запросов со стороны экономического и промышленного секторов, что представляет собой важную и актуальную задачу оперативного управления любым предприятием.

С учетом разнородности запросов работодателей, многофункциональности и многоаспектности оценок свойств выпускников 
вузов при их трудоустройстве особое внимание уделяется синтезу новых автоматизированных технологий отбора и распределения выпускников, являющихся инвариантными к своей предметной среде. Вместе с этим становятся актуальными вопросы системного моделирования в области разработки моделей и алгоритмов, направленных на структуризацию отношений и правил принятия решений, действующих на множествах потребностей работодателей и выпускников вузов. Здесь особое значение приобретает формирование проблем моделирования сценариев, описывающих поведение вузов на рынке труда, которые дают возможность сделать управление распределением выпускников максимально эффективным, а также сформировать стратегию и тактику таким образом, чтобы снизить собственные расходы и повысить уверенность в надежности качества образования.

В ходе выполнения процессов по моделированию системы выбора выпускников вузов (СВBВ) потребность в выборе последних становится актуальной лишь на этапах структурного и параметрического синтеза системы. Тогда лицом, принимающим решение (ЛПР) - работодателем, согласно определенным целям системы формируются требования к отбору качественного и количественного состава компонентов данного объекта. Известно, что данные этапы представляют собой важнейшие элементы любого процесса моделирования, поскольку качество их выполнения напрямую влияет на эффективность функционирования разрабатываемой системы. Поэтому ЛПР необходимо при реализации указанных в ходе создания модели СBBB находить не просто удовлетворительные, а оптимальные решения.

Пусть в задаче выбора выпускников вузов рассматриваемая модель оптимизации занимается решением одной ресурсной задачи.

Упрощенная математическая постановка задачи при выяснении сути проблемы выбора в СВВВ будет сформулирована таким образом $[2,3]$ :

На вход СВВВ будут поступать запросы работодателей, представляющие собой совокупность $I=\{1,2, \ldots, i, \ldots, n\}$. Задача заключается в выборе выпускников вуза, соответствующих указанным запросам. Каждому выпускнику $j$ на множестве $J=\{1,2, \ldots, j, \ldots, m\}$ поставлена в соответствие совокупность некоторых атрибутов $d_{j}(j=1, m)$.

Введем обозначение $d=\left\|d_{i j}\right\| \quad(i=\overline{1, n}$, $j=\overline{1, m})$ - это матрица, характеризующая непосредственное решение задачи выбора выпускника по указанным запросам работодателей. Характеристикой качества реализации поставленной задачи будет являться функция цели $Q(d)$, стремящаяся к минимуму. Перечень требований работодателя по формированию групп выпускников вуза будет выражен посредством множества ограничений $F: f_{k}(x) \leq \varnothing, k=\overline{1, K}$. При этом задача выбора $m$-выпускников вузов по $n$-запросам работодателей может быть сформулирована таким образом:

$$
\begin{aligned}
& Q(d) \rightarrow \text { min, } \\
& f_{k}(d) \leq 0 \leq k=\overline{1, K}, \\
& d=\left\|d_{i j}\right\|(i=\overline{1, n} ; j=\overline{1, m}) .
\end{aligned}
$$

При рассмотрении (1) с точки зрения системных позиций, можно увидеть, что в ходе решения поставленной задачи необходимо определить для СВВВ некоторое множество выпускников вузов, на котором будут достигнуты поставленные цели задачи. При этом функции данных задач будут коррелироваться с формированием такой матрицы $d$, для которой будут выполнены все ограничения модели $f_{k}(d)$, а найденное решение будет оптимальным.

На формирование матрицы $d$, ее структуры, на область анализа напрямую влияет правильное формирование исходных множеств выпускников вузов, а также их свойств.

Пусть выпускник вуза является вполне определенным, если мы имеем возможность оперировать значениями его основных свойств. При этом каждого выпускника можно охарактеризовать какими-либо проявлениями в определенных условиях, к примеру, в процессе обучения.

В настоящее время в вузах личные, общественно-значимые достижения студентов и академические успехи вне образовательного учреждения указываются в портфолио. До- 


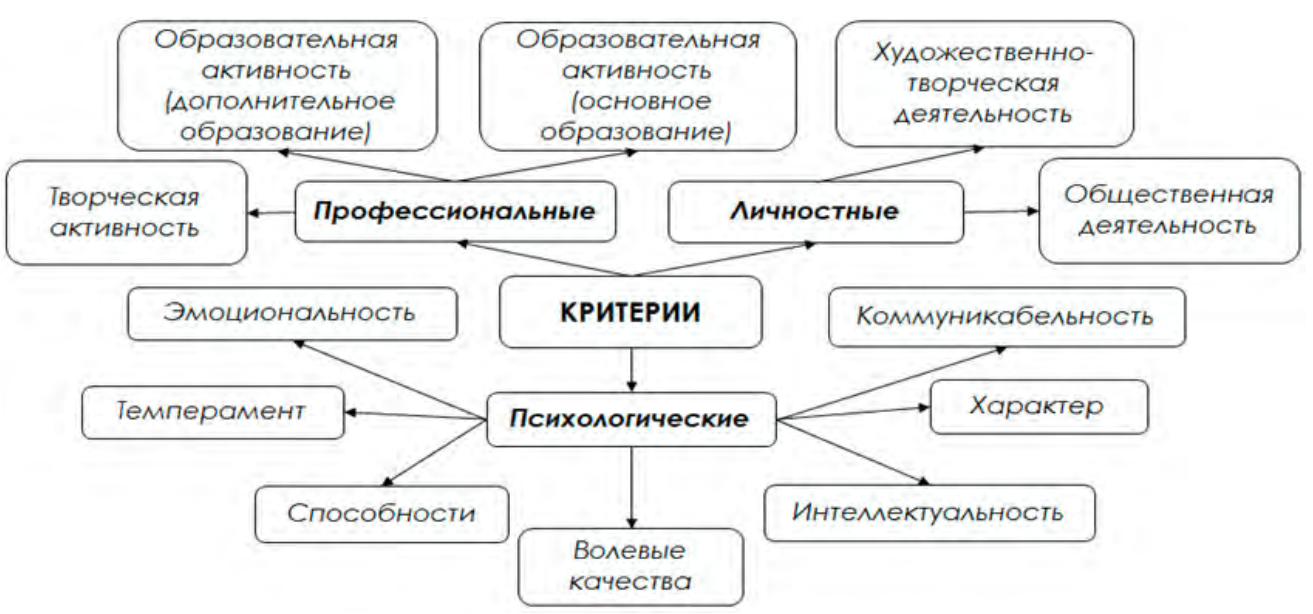

Рис. 1. Критерии оценивания выпускников вуза

стижениями принято считать результаты, которых студент добился в период обучения. Данные достижения могут подтверждаться дипломами, сертификатами, отзывами, благодарностями от руководителей практик и руководства организаций, тезисами докладов с конференций и семинаров, копией статей либо ссылок на издания со статьями студента, грамотами и прочими видами наград за призовые места в различного рода мероприятиях.

Таким образом, самостоятельно формируя портфолио, студент автоматически вырабатывает критерии оценки и отбора, беря на себя ответственность за демонстрацию своих знаний и умений. Достижения студента условно подразделяются на профессиональные, личностные, а также психологические. Более детальная оценка позволяет вычленить еще ряд подгрупп (рис. 1), в каждой из которых, в свою очередь, включен определенный набор характеристик.

Разделение на группы - важный фактор, поскольку каждая из групп имеет собственную оценку соответственно своим приоритетам. Работодателю важны профессиональные достижения, и он дает оценку студенту по совокупности всех факторов. Работодатели считают необходимым, чтобы кандидат обладал универсальностью, приверженностью профессии, обучаемостью, коммуникативностью и работоспособностью. Самооценка (оценивание студентом собственных достижений) показывает, каким образом студент оценивает себя в целом, а также конкретные составляющие собственной личности - по- ступки и действия, собственные качества, отношения, убеждения и прочее.

В результате можно говорить о способствовании портфолио студента развитию мышления, становлению критического отношения к учебе, расширению возможностей обучения и исследования. Портфолио дает возможность наглядной демонстрации развития в соответствии с прежними результатами, включения студентов в процесс понимания внешней оценки, развития их заинтересованности во внутренней самооценке, анализа, актуализации индивидуальных затруднений и поиска пути их преодоления, а также мотивации студентов на рефлексию профессиональной деятельности.

Естественно, определить количество информации о признаке выпускников вузов можно, исходя из его проявлений в определенных условиях. Лишь посредством содержательного анализа, проводимого при отборе выпускников вузов согласно целям выбора, выявления необходимой информации о выпускниках, еe вида (количественного, качественного и пр.) и свойств можно верно разработать модели принятия решения и управления потенциалом выпускника вуза (ПВВ).

Пусть требуется оценить ПВВ - конкретные атрибуты для определенной вакансии. Здесь атрибуты - свойства каждого отдельно взятого выпускника, которые выражены в его профессиональных знаниях и умениях, личностных достижениях, деловых и нравственных качествах и психологическом состоянии. 
При анализе ПВВ оцениваются качественные и количественные характеристики. Таким образом, требуется разработка профиля требований на соответствие соискателя вакансии организации. Для формирования профиля требований применяются следующие критерии:

- профессиональные критерии (уровень образования и достижения в процессе обучения);

- личностные критерии (наличие дополнительного образования, коммуникативные способности и пр.);

- психологические критерии (способность к концентрации, надежность, контактность, взаимодействие с другими членами коллектива и пр.) и т. д.

При разработке математической модели для определения соответствия выпускника вуза вакансии организации авторами предлагается комплексный подход, учитывающий критериальные оценки каждого из показателей потенциала выпускника и реализующий механизм компенсирования недостатка одного показателя существенным превышением порогового уровня другого.

Вводится индекс соответствия выпускника желаемой вакансии $J$, реализующий механизм обобщения первичных характеристик показателей в единое интегральное значение с учетом минимальных (пороговых) значений первичных характеристик показателей оценки. С другой стороны, реализуется механизм компенсирования недостатков одних первичных характеристик другими, по крайней мере, для одного показателя.

Численно определим интегральный индекс $J$ по следующим правилам: в случае совпадения значений показателей оцениваемого выпускника на должность с критериальными индексу присваивается значение единица; при превышении значений основной массы показателей оцениваемого выпускника вуза критериальных индекс считается больше единицы; в противном случае значение индекса устанавливается меньше единицы.

В результате все оцениваемые на вакансию выпускники вуза могут быть разбиты по значению индекса на ряд групп: в группу А войдут выпускники с показателями выше крите- риальных и значением индекса, большим 1; группу В составят выпускники вуза на вакансию с показателями, большинство которых превышает критериальные, а значение индекса больше или равно 1; в группу С войдут претенденты на вакансию, имеющие большинство показателей ниже, чем критериальные, а значение индекса меньше единицы. Применение индекса $J$ позволяет упрощенно принимать решения работодателю: необходимость в подробном анализе возникает чаще всего лишь в отношении выпускников с интегральным индексом меньше единицы (входят в группу C).

Пусть $k$ - количество первичных характеристик (показателей), которые используются для определения индекса, за вектор х обозначим набор значений характеристик выпускника: $x=\left(x_{1}, \ldots, x_{k}\right)^{T}$. Вектор $p=\left(p_{1}, \ldots, p_{k}\right)^{T}$ определяет соответствующие критериальные значения.

Найдем индекс $J=J(x)$ в классах таких функций:

линейная функция вида

$$
J(x)=\sum_{i=1}^{k} w_{i} \frac{x_{i}}{p_{i}},
$$

где $w_{i}$ - некоторые постоянные (веса);

линейная свертка логистических функций

$$
J(x)=c+\beta \sum_{i=1}^{k} \frac{1}{\left.1+e^{-1,7 a\left(x_{i}-p_{i}\right.}\right)},
$$

где $a, \beta, c$ - постоянные;

многомерная логистическая функция

$$
J(x)=c+\beta \frac{1}{1+e^{-1,7 \sum_{i=1}^{k} a_{i}\left(x_{i}-p_{i}\right)},},
$$

где $a_{i}, i=1 . . . k ; \beta, c$ - постоянные.

Функцией (2.1) задается линейная зависимость между значениями показателей и величиной индекса.

Линейная свертка логистических функций (2.2) основана на анализе закона распределения показателей. Основываясь на критерии Коломогорова - Смирнова, позволяющей выполнять проверку гипотезы о нормальном законе распределения случайной величины, удалось установить, что распределение показателей выполнено по нормальному закону с учетом уровня значимости, большего 0,1 . Сле- 


\section{О. В. Курипта, Ю. В. Черная, Е. В. Паршина}

довательно, логистическую функцию $J(x)$ можно рассматривать как аппроксимацию суммы интегральных функций распределения нормальной случайной величины. Функция (2.3) базируется на статических свойствах различных показателей: она является аппроксимацией функции распределения случайных величин и многомерного нормального закона распределения. Поскольку отдельные показатели распределяются по нормальному закону, их совокупность также считается случайной величиной, распределенной согласно многомерного нормального закона.

На основании функций (2.1)-(2.3) определяем модель расчета индекса соответствия статическим критериям:

Линейная модель $J 1$ :

$$
J(x)=\frac{1}{k} \sum_{i=1}^{k} \frac{x_{i}}{p_{i}}
$$

Индекс определен на основе свойства монотонности линейной функции. Данная модель отличается чрезмерной компенсацией значений негативных показателей, поскольку линейная функция является неограниченной в пространстве $R^{k}$, а значит, значительное превышение порога даже для одного показателя может привести к достаточно большому значению индекса.

Линейная модель $J 2$ : модель, имеющая ограничение компенсации значений показателей:

$$
J(x)=\frac{1}{k} \sum_{i=1}^{k}\left\{\begin{array}{l}
\frac{x_{i}}{p_{i}}, \text { если } \frac{x_{i}}{p_{i}} \\
2, \text { иначе }
\end{array}\right.
$$

Представленная модификация $J 1$ дает возможность ограничения компенсации значений показателей: большое превышение значения одного из показателей может компенсировать лишь единственное значение «провального» показателя. При этом индекс ограничивается на значение сверху $(J(x) \leq 2)$.

Логистическая модель J3:

$$
J(x)=c+\beta \sum_{i=1}^{k} \frac{1}{\left.1+e^{-1,7\left(x_{i}-p_{i}\right.}\right)} .
$$

Применение логистических функций (2.6) основывается на анализе закона распределения показателей. Здесь расчеты могут рассматриваться как приближенная оценка вероятности для суммы случайной величины, распределенной согласно нормальному закону.

Параметры модели выбираются, исходя из следующих условий:

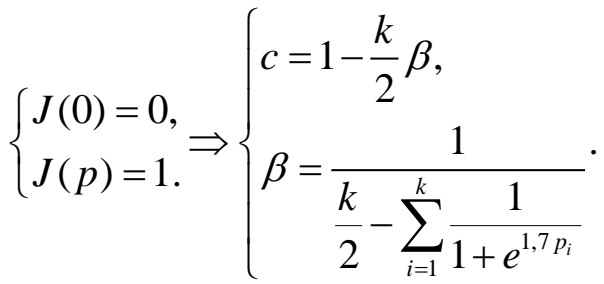

Предложенная модель монотонна по $x$ и отличается довольно быстрым ростом индекса при возрастании значения переменной.

Логистическая модель J4 (с ограничением компенсаций значения показателей):

$$
\begin{gathered}
J(x)=\beta \sum_{i=1}^{k} \frac{1}{\left.1+e^{-1,7 a\left(x_{i}-p_{i}\right.}\right)} \\
\left\{\begin{array} { l } 
{ J ( p ) = 1 } \\
{ J ( 2 p ) = 2 - \delta }
\end{array} \Rightarrow \left\{\begin{array}{l}
\beta=\frac{2}{k}, \\
a=\frac{\ln \left(\frac{2}{\delta}-1\right)}{1,7 \max p_{i}}
\end{array}\right.\right.
\end{gathered}
$$

где $\delta>0$ - достаточно малый параметр.

Данная модель представляет модификацию предыдущей, позволяя снизить компенсацию значений показателей посредством ввода дополнительных ограничений на значение индекса в точке $2 p$ (если наблюдается двукратное превышение пороговых значений по показателям, а индекс не превышает 2).

Многомерная модель $J 5$ :

$$
J(x)=c+\beta \frac{1}{1+e^{-1,7 \sum_{i=1}^{k}\left(x_{i}-p_{i}\right)} .}
$$

Данная модель является интегральной, основанной на многомерном нормальном законе распределения показателей. Вычисление параметров показателей осуществляется согласно следующим условиям:

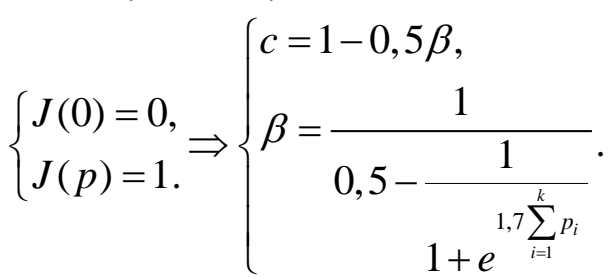


Указанная модель отличается медленным ограниченным ростом значений и «естественным» способом агрегирования первичных показателей, который определяется статистическими свойствами имеющихся показателей и математическим видом функций.

В приведенных моделях применяется линейная свертка с весовыми коэффициентами. Одинаковая важность всех показателей предполагает выбор равных коэффициентов. При этом характеристики внутри отдельно взятого показателя могут быть с разными весовыми коэффициентами, в то время как показатели в целом включены в сумму с равными весами. Осмысленный выбор весов можно провести экспертным путем.

Указанные математические модели дают возможность правильной и достоверной оценки отдельно взятого студента на соответствие предлагаемой должности, что позволяет облегчить работодателю процедуру принятия решения при приеме кандидата на работу.

Согласно результатам деятельности различных предприятий и накопленному опыту их работы с трудовыми ресурсами, решаю-

Курипта Оксана Валерьевна - канд. техн. наук, доцент кафедры информационных технологий и автоматизированного проектирования в строительстве ВГТУ.

Тел.: (910) 344-3199

Черная Юлия Викторовна - магистрант направления подготовки «Информационные системы и технологии» ВГТУ кафедры информационных технологий и автоматизированного проектирования в строительстве ВГТУ.

Тел.: (920) 467-1015

E-mail: juli1574@mail.ru

Паршина Елена Владимировна - старший преподаватель кафедры информационных технологий в экономике Воронежского филиала РЭУ им. Г. В. Плеханова.

Тел.: (473) 239-0763 щую роль при создании конкурентоспособного предприятия играет правильное формирование производственных коллективов, отличающихся высоким качеством кадрового потенциала.

\section{СПИСОК ЛИТЕРАТУРЫ}

1. Черная Ю. В., Никитина А. Л. Оценка качества знаний студентов с применением методики на основе Раш-анализа // Научный вестник Воронежского государственного архитектурно-строительного университета. Серия: Информационные технологии в строительных, социальных и экономических системах. - 2017. - №1(9).

2. Курипта О. В., Сербулов Ю. С. Модели оптимального управления трудовыми ресурсами организации // Моделирование систем и информационные технологии: Межвуз. сб. науч. тр. - Воронеж: Научная книга, 2006. Вып. 3. - Ч. II - С. 67-74.

3. Курипта О. В., Сербулов Ю. С. Коровина О. В. Модели мониторинга уровня развития вуза// Инженерная физика. - М. : НАУЧ. ТЕХЛИТИЗДАТ, 2010. - № 4. - С 9-11.

Kuripta Oksana Valerievna - candidate of technical Sciences, associate Professor, Department of information technology and computer-aided design in the construction of VGTU.

Tel.: (910) 344-3199

Chernaya Yuliya Viktorovna - graduate student of specialty «Information systems and technologies», Department of information technology and computer-aided design in the construction of VGTU.

Tel.: (920) 467-1015

E-mail: juli1574@mail.ru

Parshina Elena Vladimirovna - the senior teacher of chair of information technologies in the economy of the Voronezh branch of REU them. G. V. Plekhanov.

Tel.: (473) 239-0763 